\title{
Order in the pericentriolar material
}

The centrosome is the main microtubule-organizing centre in animal cells and has many crucial functions, including the assembly of the mitotic spindle and establishing the axis of cell division. It comprises a pair of barrel-shaped centrioles that are surrounded by pericentriolar material (PCM). Although the PCM is normally described as an electrondense amorphous protein cloud, four studies now reveal that it is in fact highly organized.

Lawo et al. and Sonnen et al. visualized the localization of PCM components in human cells using three-dimensional structured illumination microscopy (3D-SIM). They observed that, with the exception of pericentrin (PCNT), PCM proteins are organized in a layered manner and form cylinders that surround the mother centriole during interphase. PCNT, which is one of many proteins implicated in the recruitment and/or attachment of $\gamma$-tubulin ring complexes (that mediate microtubule nucleation), was found to adopt an elongated conformation spanning the width of the PCM, with the carboxyl terminus positioned near the centriole and the amino terminus extending out to the periphery. Importantly, Lawo et al. found that the organization of the PCM was disrupted when cells were depleted of PCNT, which suggests that this protein functions as a scaffold for PCM assembly.

Similarly, Mennella et al. and $\mathrm{Fu}$ and Glover used 3D-SIM to quantitatively map the architecture of the PCM in Drosophila melanogaster cells. They found that the PCM is organized into two main structural domains with distinct molecular composition: an inner layer that is juxtaposed to the centriole wall; and an outer layer that extends further away from the centrioles.

By combining 3D-SIM with volume averaging, Mennella et al. were able to map the position of pericentrin-like protein (PLP) domains with nanometre precision, showing that PLP forms elongated structures oriented radial to the centriole wall, with the $\mathrm{C}$ terminus positioned towards the centriole. Stochastic optical reconstruction microscopy (STROM), which provides higher resolution than SIM, further revealed that PLP has a quasininefold symmetry and thus extends the centriole symmetry into the PCM matrix. Crucially, RNAi experiments showed that, like PCNT in human cells, these PLP fibrils function as a scaffold for the $3 \mathrm{D}$ assembly of the outer PCM.

The PCM assembles in the vicinity of centrioles during mitosis and expands during centrosome maturation, so Sonnen et al. and $\mathrm{Fu}$ and Glover asked whether protein localization in the PCM changes at mitotic entry. Interestingly, Sonnen et al. observed that, instead of the layered organization seen in interphase, during mitosis PCM proteins form networks with interspersed $\gamma$-tubulin. Moreover, both groups show how centriolar proteins contribute to the expansion of the PCM. For example, they observed that Centrosomin (CNN) and $\gamma$-tubulin are first recruited to an inner PCM layer prior to expanding into the outer part of the PCM together with
Spindle-defective protein 2 (SPD2). Centriolar Polo-like kinase 1 (PLK1) lies at the head of the hierarchy, whereas $\mathrm{CNN}$ is required for SPD2 to localize to the PCM and together they recruit $\gamma$-tubulin.

At each cell cycle, centrioles duplicate, and all four studies examined how the localization of the PCM and centriolar components relates to the assembly of nascent daughter centrioles (which appear perpendicular to the mother centrioles). Interestingly, they observed that PCNT and PLP around the mother centrioles form a gap at the site where the daughter centriole emerges. Furthermore, Sonnen et al. also report that centriolar PLK4 constitutes an early marker for this site of nascent centriole formation.

These four studies show that the PCM has a conserved ordered structure in which proteins occupy distinct domains, and also suggest that these locations have functional implications for the assembly and maturation of the centrosome. Importantly, they highlight the power of new microscopy methods, paving the way for future investigations.

Kim Baumann

ORIGINAL RESEARCH PAPERS Lawo, S. et al. Subdiffraction imaging of centrosomes reveals higher-order organizational features of pericentriolar material. Nature Cell Biol. 21 Oct 2012 (doi:10.1038/ncb2591)|Sonnen, K. F.et al. 3D-structured illumination microscoscopy provides novel insight into architecture of human centrosome. Biol. Open 17 Aug 2012(doi:10.1042) bio.20122337)| Mennella, V. et al. Subdiffractionresolution fluorescence microscopy reveals a domain of the centrosome critical for pericentriolar material organization. Nature Cell Biol. 21 Oct 2012 (doi:10.1038/ncb2597)| Fu, J. \& Glover, D. M. Structured illumination of the interface between centriole and peri-centriolar material. Open Biol. 2, 120104 (2012) 